\title{
Towards reactor neutrino applied physics
}

\author{
M. Fallot ${ }^{1, \mathrm{a}}$, S. Cormon ${ }^{1}$, M. Cribier ${ }^{2}$, L. Giot ${ }^{1}$, B. Guillon ${ }^{1}$, T. Lasserre ${ }^{2}$, A. Letourneau ${ }^{3}$, D. Lhuillier ${ }^{3}$, and J. Martino ${ }^{1}$ \\ 1 SUBATECH - CNRS/IN2P3, Université de Nantes, École des Mines de Nantes, Rue A. Kastler, BP. 20722, 44307 Nantes, France \\ 2 CEA/DAPNIA/SPP - APC, 10 rue A. Domon et L. Duquet, 75205 Paris Cedex 13, France \\ ${ }^{3}$ CEA/DAPNIA/SPhN Saclay, 91191 Gif-sur-Yvette Cedex, France
}

\begin{abstract}
Nuclear power plants are intense sources of antineutrinos. Their energy spectrum and emitted flux depend on the composition of the nuclear fuel and on the thermal power of the reactor. These properties led to potential applications of neutrino physics: they could be used to non intrusively monitor a nuclear reactor. To this purpose, a better knowledge of the antineutrino energy spectra arising from uranium and plutonium isotope fission is necessary. In these proceedings we relate about on-going simulation efforts aiming at reducing the errors associated to these spectra. The generic tools under development and presented below will allow to perform scenario studies on the feasibility of using antineutrinos to measure thermal power and to test to which precision the fuel composition can be deduced using these particles.
\end{abstract}

\section{Introduction}

Neutrino properties have been extensively studied since their discovery by F. Reines and C. Cowan in 1957 near the Savannah River reactor (USA) [1,2]. In the 70's was born the idea that neutrino properties could also be useful for applications linked with nuclear power plants [3]. Indeed, the numerous electronic antineutrinos emitted by a nuclear power plant arise mainly from the fission of uranium and plutonium isotopes $\left({ }^{235} \mathrm{U},{ }^{238} \mathrm{U},{ }^{239} \mathrm{Pu},{ }^{241} \mathrm{Pu}\right)$. Their fission characteristics are different (cf. table 1). The fission products are neutronrich nuclei and undergo beta decay, emitting antineutrinos. The subsequent antineutrino energy spectra and the mean number of emitted antineutrinos reflect the fission product distributions. As a consequence, the energy spectrum coming from a reactor reflects the fuel composition and the neutrino flux is directly proportional to its thermal power for a fixed composition. Thus a typical nuclear power reactor of $900 \mathrm{MWe}$ emits $\simeq 2 \cdot 10^{20} \overline{v_{e}} / \mathrm{s}$ (above $1.8 \mathrm{MeV}$ ). A change of the antineutrino spectrum shape with the burn-up (build-up of ${ }^{239} \mathrm{Pu}$ and consumption of ${ }^{235} \mathrm{U}$ ) has already been observed in previous neutrino experiments $[4,5]$ and in a recent experiment at the San Onofre power plant [6].

Antineutrinos, thanks to their tiny interaction cross section, escape without interacting and their measurement provide a clean image of the whole reactor core. The time exposure of a few cubic meter detector placed at 10-30 m from the core ranges from a few hours to a few days depending on the requested resolution. Hence antineutrinos could constitute an interesting safeguards tool for the International Atomic Energy Agency (IAEA), allowing to make remote, non-intrusive measurements of plutonium content in reactors. The Agency has asked to its member states to perform a feasibility study. The small magnitude of the seeked signal requires a carefull study. Huber and Schwetz performed a sensitivity study, considering the actual knowledge on the antineutrino flux from $\mathrm{U}$ and $\mathrm{Pu}$ isotopes [7]. To reach the

${ }^{a}$ Presenting author, e-mail: fallot@subatech.in2p3.fr required accuracy, a reduction of the uncertainties on the antineutrino flux by a factor 3 to 10 should be performed. They quote also that without any improvement, a precision of $3 \%$ on the daily thermal power measurement could already be reached in reasonable experimental conditions.

Table 1. Main characteristics of antineutrinos originating from ${ }^{235} \mathrm{U}$ and ${ }^{239} \mathrm{Pu}$ fission.

\begin{tabular}{lll}
\hline & ${ }^{235} \mathrm{U}$ & ${ }^{239} \mathrm{Pu}$ \\
\hline released energy per fission & $201.7 \mathrm{MeV}$ & $210.0 \mathrm{MeV}$ \\
Mean energy of $\overline{v_{e}}$ & $2.94 \mathrm{MeV}$ & $2.84 \mathrm{MeV}$ \\
$\overline{v_{e}}$ per fission $\geq 1.8 \mathrm{MeV}$ & 1.92 & 1.45 \\
average inter. cross section & $\simeq 3.2 \cdot 10^{-43} \mathrm{~cm}^{2}$ & $\simeq 2.8 \cdot 10^{-43} \mathrm{~cm}^{2}$ \\
\hline
\end{tabular}

In these proceedings, we report on studies aiming at a better determination of the antineutrino energy spectrum from nuclear power plants for applied neutrino physics, but also for experiments studying the fundamental properties of these particles such as Double Chooz [8]. This experiment will seek for a non-zero $\theta_{13}$ mixing angle with two identical detectors placed near the Chooz nuclear power plant, at $280 \mathrm{~m}$ and $1050 \mathrm{~m}$ from the reactors, respectively. Antineutrinos are detected via their beta inverse reaction $\overline{v_{e}}+p \rightarrow e^{+}+n$, threshold: $1.8 \mathrm{MeV}$. During the first phase of the experiment, only the far detector will be running and our work will be used as an accurate normalisation of the experiment. For these reasons the work presented below is the result of a close collaboration within the Double Chooz experiment.

In the following, we will first describe the previous studies led in the 80's in order to determine antineutrino energy spectra from the main fissile isotopes composing the nuclear fuel. Then we will present our strategy to improve the current knowledge. In particular, the fission product decay data available in the nuclear databases have considerably increased over the last twenty years. Taking into account these new data is mandatory in order to determine the future additional lab tests 
and theoretical studies that should be performed to make the antineutrino applications possible. A preliminary evaluation of the errors attached to these spectra will be presented. We will finally conclude and present our outlooks.

\section{Previous estimations of antineutrino spectra}

Several approaches were followed to access the antineutrino spectra of fissile isotopes. Schreckenbach et al. measured integral $\beta$-spectra from ${ }^{235} \mathrm{U},{ }^{239,241} \mathrm{Pu}$ at the Institut Laue Langevin, Grenoble [9]. They performed very precise measurements up to $8 \mathrm{MeV}$. In order to convert these $\beta$-spectra into antineutrino ones, the conservation of energy should be applied, using each individual end-point of the beta branches composing the spectra. As Schreckenbach et al. did not have these informations, they fitted each integral beta spectrum with a set of 30 fictive beta branches. This conversion procedure induces an important uncertainty on the shape of the antineutrino spectra.

In order to solve this problem, Tengblad et al. measured the individual beta spectra of 111 fission products [11]. They conjugated these $\beta$-spectra with decay properties of 265 other fission products from nuclear databases and built the resulting antineutrino spectra for ${ }^{235,238} \mathrm{U}$, and ${ }^{239} \mathrm{Pu}$. Important errors were attached to the obtained global spectra $(5 \%$ at $4 \mathrm{MeV}$, $11 \%$ at $5 \mathrm{MeV}$ and $20 \%$ at $8 \mathrm{MeV}$ ) and a notable disagreement was observed with the results of Schreckenbach et al. Up to now their measured spectra remain the most precise ones, and were used in fundamental neutrino experiments such as Bugey and Chooz [5,10]. C. Bemporad [12] claimed recently that unknown decays contribute as much as $25 \%$ of the antineutrinos at energies higher than $4 \mathrm{MeV}$. This could explain the shape discrepancy between the spectra obtained by Tengblad and Schreckenbach.

Several strategies can be adopted in order to reduce the error bars on the antineutrino energy spectra of fissile isotopes. The numerous informations on the fission products contained nowadays in databases can be used directly to build branch by branch the integral beta spectra and convert them into antineutrino ones without any ambiguity. We will use this latest approach, which was already adopted by Vogel et al. more than 25 years ago [13]. Moreover, the knowledge of individual contributions of fission products allows to simulate the precise evolution with time of the antineutrino spectrum emitted by a nuclear reactor and to simulate the fuel burnup, when coupled to a precise simulation of the core. We are therefore developing generic simulation tools that can be used to simulate the antineutrino spectrum for any reactor type. The following section is devoted to their description and to the first results we obtain.

\section{Towards a better determination of the antineutrino spectrum from reactors}

Subatech-Nantes and CEA/DAPNIA/SPhN started a simulation work using the widely used particle transport code MCNPX [14], coupled with an evolution code solving the
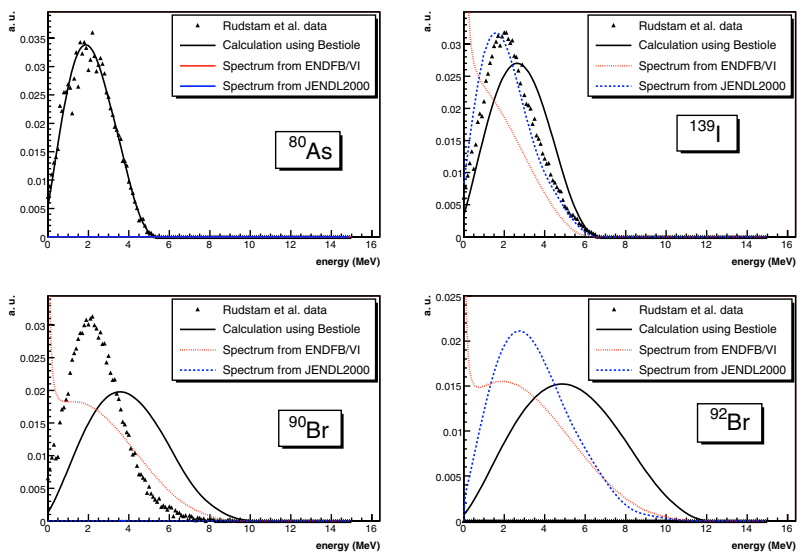

Fig. 1. Black triangles: experimental $\beta$-spectra of ${ }^{80} \mathrm{As},{ }^{139} \mathrm{I},{ }^{90,92} \mathrm{Br}$ taken from [20]. Black line: result of the BESTIOLE calculation. Blue dashed line: continuous spectra extracted from JENDL 2000 fission product decay data. Red dotted lines: continuous spectra found in ENSDF/B-VI database.

Bateman equations for the fission products within a package called MURE (MCNP Utility for Reactor Evolution) [15]. The evolution in time of the isotopic composition of the core is then only driven by the initial fuel composition and the input of the thermal power given by the operators of the power plant. This package, developed by the LPSC Grenoble and IPN Orsay labs, offers a set of tools, interfaced with MCNP or MCNPX, that allows to define the geometry of a reactor core in a very simple way, and compiles a set of nuclear data used in the evolution part [16]. 3834 nuclei can be possibly considered. MURE is perfectly adapted to take into account the physics of a reactor core, especially the neutronics as neutron capture in accumulated fission products give small but non negligible local distortions of the total energy spectrum, as quoted by V. Kopeikin et al. [17]. It will constitute an efficient tool for non-proliferation and thermal power scenario studies.

This code, giving the total amount of each fission product produced in the experimental conditions, is coupled to existing databases containing beta decay properties of a large number of fission products. The ENSDF database [16] contains for each branch (more than 10000!), when it is known, endpoints, branching ratios, and spin/parities of $\beta$-transitions. A package called BESTIOLE has been developed to read these informations and give them as an input to a code inspired from the BETA-S code from Oak Ridge [18], computing the $\beta$-spectrum shapes, taking into account the type of the transition. Allowed and forbidden unique transitions up to the 3rd degree can be computed precisely [19]. Forbidden nonunique transitions cannot be simulated and approximations are made; for an $\mathrm{n}^{\text {th }}$ forbidden non-unique transition the shape of the corresponding $(n-1)^{\text {th }}$ unique transition is taken. Resulting individual $\beta$-spectra are compared with the experimental spectra measured by Tengblad et al. [20]. Examples are shown in figure 1. Experimental spectra for allowed and forbidden unique transitions are very well reproduced by the BESTIOLE calculations (see ${ }^{136} \mathrm{I}$ and ${ }^{80} \mathrm{As}$ spectra for instance). When non-unique transitions contribute importantly the simulation deviates significantly from the data (see $\mathrm{Br}$ isotopes). 


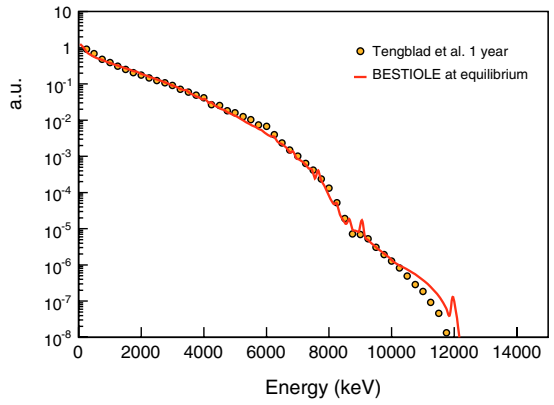

Fig. 2. Comparison of beta spectra from ${ }^{235} \mathrm{U}$ fission. Red points: from [11], extrapolation to 1 year irradiation. Red line: simulation at equilibrium using cumulative yields, BESTIOLE and 63 experimental spectra from [20] (see text).

The global ${ }^{235} \mathrm{U}$ beta spectrum has been simulated using the MURE and BESTIOLE packages. For a non negligible amount of very neutron-rich fission products no data can be found in the ENSDF database. In this case we have computed, in a first attempt, the associated $\beta$-spectra assuming only one allowed $\beta$-transition towards the ground state of the daughter nucleus. We call this approximation "Qbeta approximation". This approximation is very crude as very neutron-rich fission products usually exhibit important $\beta$-branches towards excited states in the daughter nucleus and important $\beta$-delayed neutron emissions. This leads to an overestimation of the high energy part of the fission product beta spectra. As a first attempt, we computed the integral $\beta$-spectrum shown in figure 2, summing all individual branches for the 375 nuclei selected by Tengblad et al. using cumulative yields from ENDF. $\beta$ spectra from BESTIOLE and 63 experimental $\beta$-spectra taken from ref. [20] that were not well reproduced by BESTIOLE are taken into account. Comparing the result with the integral spectrum from [11] in figure 2, the obtained agreement is very good. This good result is a validation of the BESTIOLE package. We then computed the integral beta spectrum from ${ }^{235} \mathrm{U}$ fission including more nuclei in the calculation of the spectra (more than 900 fission products). The shape of the spectrum is significantly changed, especially at high energy. The result is compared with Schreckenbach's data in figure 3 (left) for a $1.5 \mathrm{~d}$ irradiation time, computed using the MURE code. Shorter irradiation times enhance the role played by short half-life nuclei (often exhibiting high energy end-points). These effects are illustrated by the large overestimation of the data by the simulation above $5 \mathrm{MeV}$. Among the considered fission products, a large fraction is composed of exotic nuclei which are very difficult to study experimentally and for which no or partial informations are available in the databases. The Qbeta approximation was therefore applied for these nuclei. First forbidden non-unique transitions contribute also significantly to the global spectrum, underlining the necessity of measuring the beta energy spectrum shapes for such transitions. Focussing on the energy range from 2 to $5 \mathrm{MeV}$ and normalising the simulation on the data in this interval, a very good agreement with Schreckenbach's beta spectrum is found. This confirms the importance of shorter half-life nuclei in explaining the discrepancies between Schreckenbach's and Tengblad's measurements.
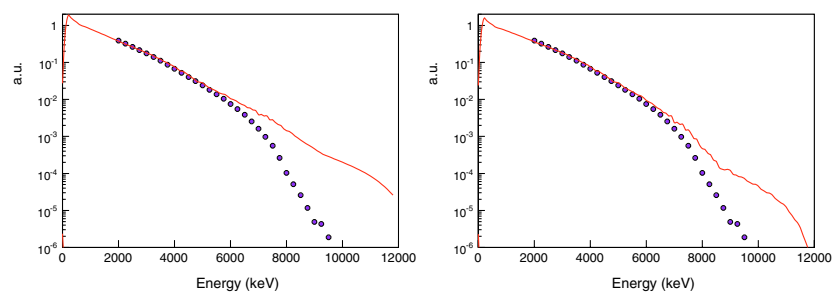

Fig. 3. Simulations (line) compared with data from [9] (points) for a $1.5 \mathrm{~d}$ irradiation. Left: Qbeta approximation was used. Right: continuous spectra from ENDFBVI, JENDL and JEFF-3.1 were included.

To improve the simulation and replace the Qbeta approximation for these exotic nuclei, we coupled the MURE package to other databases that contain continuous beta spectra for very neutron rich nuclei computed with the Gross Theory [21]: ENDF/B-VI, JENDL and JEFF-3.1. This study is in progress, and we will present here only a few preliminary comparisons between experimental individual $\beta$-spectra coming from Rudstam's tables, and the spectra contained in ENDFBVI and JENDL2000 databases. These comparisons allow to get an idea of the predictive power of the Gross Theory spectra contained in the databases when no experimental data exist. In figure 1, continuous spectra, both using the Gross Theory, are shown when they are present in the databases for the above considered nuclei. One can already notice that the shape of the spectra from JENDL and from ENDF are very different. Reactor safety simulations for which these data have usually been developed require only the mean $\beta$-energy. This preliminary study will be refined and recent models based on the Gross Theory, developed to take into account structure effects, will be also tested [22]. In figure 3, the result of the inclusion of the Gross Theory spectra on the global beta spectrum from ${ }^{235} \mathrm{U}$ is presented. The "Qbeta approximation" has been replaced by the Gross Theory spectra that can be found in ENDF/B-VI in priority, JENDL when the nucleus is not present in ENDF/B-VI and then JEFF-3.1. The high energy part is much lower than when using the "Qbeta approximation", but still overestimates Schreckenbach's data. In order to evaluate the role played by the unknown branches in the databases, we computed the respective percentages of the number of electrons per $1 \mathrm{MeV}$ bin of each contribution (cf. fig. 4). The contribution of the unknown beta branches variates strongly depending on the assumption made for these branches. Even if the inclusion of the Gross Theory spectra does not solve fully the high energy discrepancy, we can see that our simulation of the spectrum from $2 \mathrm{MeV}$ up to $5 \mathrm{MeV}$ is rather robust and well under control when looking at figure 4 . This is very encouraging for fundamental neutrino experiments which are mostly concerned in this energy range.

\section{Preliminary evaluation of the actual uncertainties on the ${ }^{235} \mathrm{U}$ antineutrino spectrum}

In our approach, all sources of uncertainties on the total antineutrino spectrum are carefully propagated and the contribution of any isotope to the total spectrum versus time and energy is known. The effect of the uncertainty of the 


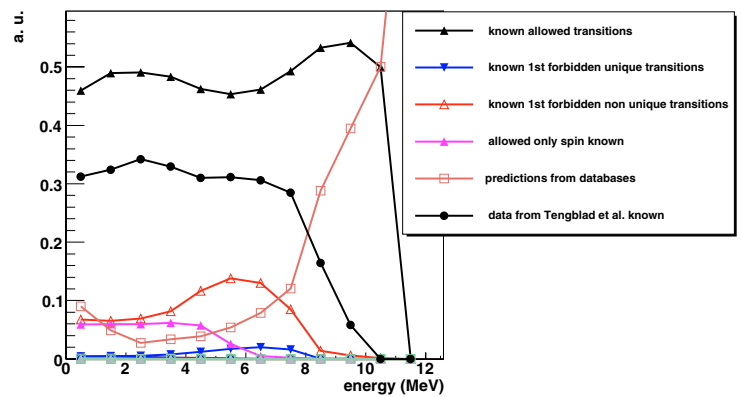

Fig. 4. Bin per bin percentages of the number of electrons coming from the contributions explicited in the legend.
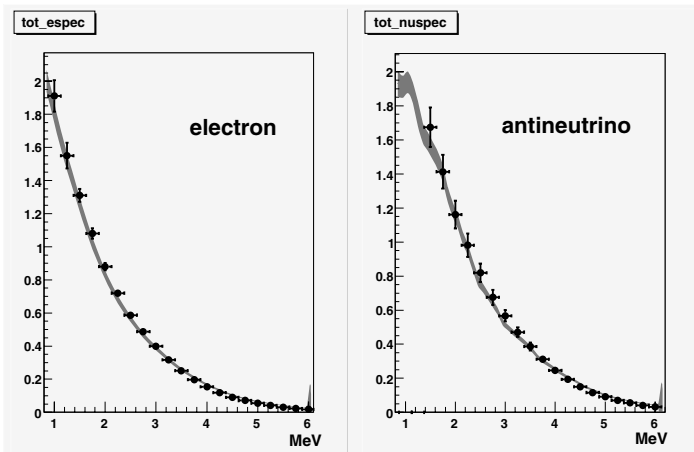

Fig. 5. Our simulations of the $\beta$-spectrum from ${ }^{235} \mathrm{U}$ beta spectrum (left) and antineutrino spectrum (right) with the computed errors, compared with the spectra from [9].

parameters (end-points, branching ratios) on the associated electron and antineutrino spectra is computed in all energy bins and presented in figure 5. Applying energy conservation branch per branch, the corresponding antineutrino spectrum is obtained (fig. 5, right). One can see that the errors quoted on the antineutrino spectrum bins are usually smaller than Schreckenbach ones. At this stage of our simulation, the error on the shape of $\mathrm{n}^{\text {th }}$ non unique transitions is estimated from the variation in shape of $n^{\text {th }}$ and $(n \pm 1)^{\text {th }}$ unique transitions. This amounts to, at most, a $\pm 2.5 \%$ error per bin, according to this preliminary evaluation. The estimation of the error associated with the conversion procedure of the 63 experimental spectra included from [20] is still in progress. The last sources of uncertainty to be included are the fission yields and the halflives data used by the evolution code. The propagation of these errors through the Bateman evolution equations is not trivial and will be treated numerically. This work is in progress, as well as the treatment of correlations that we expect large between contiguous energy bins and that should significantly increase the sensitivity to any shape distortion of the spectrum.

\section{Conclusion and outlooks}

In conclusion, a detailed simulation of the antineutrino spectrum emitted by a reactor core is being developed for neutrino applied physics purposes but also for fundamental neutrino experiments such as Double Chooz. The code MCNP Utility for Reactor Evolution (MURE) has been adapted to compute the amount of all $\beta$-emitters produced during the operation of a nuclear power plant. The emitted antineutrino energy spectrum is then built from each individual fission product, using an accurate modelling of all $\beta$-branches indexed in nuclear databases. In this approach, all sources of error on the total antineutrino spectrum are carefully propagated and the contribution of any isotope to the total spectrum versus time and energy is known. The developed tools are perfectly adapted to perform non-proliferation and thermal power scenario studies. The comparison of our simulation results with total $\beta$-spectra measured at the ILL shows an overestimation of the high energy part of the $\beta$-spectrum from ${ }^{235} \mathrm{U}$ due to the contribution of unknown $\beta$-branches of exotic nuclei. The inclusion of Gross Theory spectra from the JENDL and ENDFB-VI databases is under test but their comparison to known fission product beta spectra does not seem satisfactory. Refined Gross Theory models should be tested in the near future. The high energy part problem could also originate partially from what is called the "Pandemonium effect" [23]. Indeed in the databases some experimental information are biased due to the difficulty of measuring high energy $\gamma$-rays or low energy $\gamma$-cascades. These features will be studied carefully in the near future with the help of the TAGS collaboration [23]. When the error analysis will be complete, a list of nuclei which decay properties and yields have to be better determined experimentally will be established.

\section{References}

1. C.L. Cowan Jr., F. Reines et al., Science 124, 103 (1956).

2. S. Fukuda et al. (Super-Kamiokande coll.), Phys. Lett. B 539, 179 (2002); K. Eguchi et al. (Kamland coll.), Phys. Rev. Lett. 90, 021802 (2003).

3. L.A. Mikaelian, Proc. Int. Conf. Neutrino-77, Vol. 2, p. 383.

4. Klimov et al., At. Energy 76-2, 123 (1994).

5. Y. Declais et al., Nucl. Phys. B 434, 503 (1995).

6. A. Bernstein et al., J. Appl. Phys. 91, 4672 (2002).

7. P. Huber, T. Schwetz, Phys. Rev. D 70, 053011 (2004).

8. F. Ardellier et al., Double-Chooz coll., arXiv:hep-ex/0606025.

9. A.A. Hahn et al., Phys. Lett. B 218, 365 (1989).

10. M. Appollonio et al., Eur. Phys. J. C 27, 331 (2003).

11. O. Tengblad et al., Nucl. Phys. A 503, 136 (1989).

12. Bemporad et al., Rev. Mod. Phys. 74, (2002).

13. P. Vogel et al., Phys. Rev. C 24, 1543 (1981).

14. J.S. Hendricks et al., Monte Carlo N-Particle eXtended, LA-UR05-2675.

15. O. Meplan et al., MURE: MCNP Utility for Reactor Evolution. Proceedings of the ENC 2005 (CD-Rom) 1 (2005).

16. ENDF/B-VI.8, JENDL-3.3, JEFF-3.1, Evaluated nuclear reaction datafiles and Evaluated Nuclear Structure Data File, from the NNDC, http://www3.nndc.bnl.gov/index.jsp Fission yields from T.R. England, B.F. Rider, ENDF-349, LA-UR-943106.

17. V. Kopeikin, L. Mikaelyan, V. Sinev, Phys. At. Nucl. 67, 1963 (2004).

18. Oak Ridge Report COG-96-33-I RC-1564.

19. H. Behrens, L. Szybisz, Shapes of $\beta$-spectra, ZAED, Physics Data 6-1.

20. G. Rudstam et al., At. Data Nucl. Data Tables 45, 239 (1990).

21. K. Takahashi, M. Yamada, Prog. Theo. Phys. 41, 6 (1969).

22. H. Nakata, T. Tachibana, M. Yamada, Nucl. Phys. A 521 (1997).

23. Private comm. from A. Algora, J.L. Tain (these proceedings). 